\title{
Enquête de l'IEA sur le niveau en lecture - II
}

Le cas de la France

\section{Émilie Barrier}

\section{(2) OpenEdition}

Journals

Édition électronique

URL : http://journals.openedition.org/ries/4260

DOI : $10.4000 /$ ries.4260

ISSN : 2261-4265

Éditeur

Centre international d'études pédagogiques

Édition imprimée

Date de publication : 1 juin 1994

Pagination : 49-53

ISSN : $1254-4590$

Référence électronique

Émilie Barrier, «Enquête de l'IEA sur le niveau en lecture - II 》, Revue internationale d'éducation de Sèvres [En ligne], 02 | 1994, mis en ligne le 17 avril 2015, consulté le 19 avril 2019. URL : http:// journals.openedition.org/ries/4260; DOI : 10.4000/ries.4260

Ce document a été généré automatiquement le 19 avril 2019

(c) Tous droits réservés 


\title{
Enquête de l'IEA sur le niveau en lecture - II
}

\author{
Le cas de la France
}

Émilie Barrier

1 Les résultats de l'enquête internationale sur le niveau en lecture qui s'est déroulée au printemps 1991 dans une trentaine de pays des deux hémisphères, dont tous les pays de l'Union européenne (sauf l'Angleterre et le Luxembourg), contredisent ce qui est communément admis aujourd'hui en France - un fort taux d'illettrisme à l'entrée en sixième - mais aussi posent des questions sur la façon dont est traité ce problème lorsqu'il existe, particulièrement à l'école primaire.

2 Rappelons que cette enquête a été effectuée en milieu scolaire, dans les classes où la majorité des élèves avait entre 9 ans et 9 ans 11 mois, ou entre 13 ans et 13 ans 11 mois, au moment des tests. Ces élèves se trouvent, en France, dans des classes de CM1 ou de troisième des collèges. En CM1 et en troisième des élèves plus âgés que la normale, parce qu'ils ont redoublé, sont assez nombreux, ce qui se traduit par une diminution de la moyenne générale (les redoublants ayant traditionnellement de moins bons résultats).

3 L'accent a donc été mis sur les formes écrites qui sont fréquemment utilisées dans une société organisée (journaux, cartes, graphiques, notices, circulaires aussi bien que livres et revues) avec trois types de textes: les narrations, textes linéaires qui racontent une histoire et impliquent plus ou moins émotionnellement le lecteur; les exposés qui décrivent, expliquent, donnent des informations factuelles sur des thèmes scientifiques par exemple, la lecture de l'âge d'un arbre ou les utilisations du laser; enfin les documents qui sont des informations structurées sous forme de tableaux, cartes, listes d'instructions, tables des matières où les élèves ont à localiser des informations plutôt qu'à lire chaque mot d'un texte.

4 Le niveau d'un élève est mesuré dans chacun des trois domaines (narrations, exposés, documents), sur une même échelle internationale dont la moyenne est de 500 et l'écarttype de 100. 
Que peut-on dire des résultats en termes de comparaison? La France se trouve parmi les pays en tête avec une moyenne supérieure à la moyenne internationale.

Pour la lecture des classes correspondant au CM1, la France n'est précédée que par la Finlande, les États-Unis et la Suède. Elle fait jeu égal avec l'Italie et la Nouvelle-Zélande et précède largement les pays européens dont l'Allemagne - une différence de 4 à 6 points entre les pays n'étant pas significative. En CM1, en particulier, les élèves qui ne parlent pas français à la maison ont de meilleurs résultats que les élèves néerlandophones dans leur propre pays.

Moyennes nationales pour les élèves de CM1 (ou classes équivalentes)

\begin{tabular}{|l|c|c|c|c|}
\hline \multicolumn{1}{|c|}{ Pays } & $\begin{array}{c}\text { Moyenne } \\
\text { générale }\end{array}$ & Narrations & Exposés & Documents \\
\hline Finlande & 569 & 568 & 569 & 569 \\
USA & 547 & 553 & 538 & 550 \\
Suède & 539 & 536 & 542 & 539 \\
France & 531 & 532 & 533 & 527 \\
Italie & 529 & 533 & 538 & 517 \\
Nouvelle-Zélande & 528 & 534 & 531 & 521 \\
Norvège & 524 & 525 & 528 & 519 \\
Islande & 518 & 518 & 517 & 519 \\
Hong Kong & 517 & 494 & 503 & 554 \\
Singapour & 515 & 521 & 519 & 504 \\
Suisse & 511 & 506 & 507 & 522 \\
Irlande & 509 & 518 & 514 & 495 \\
Belgique francoph. & 507 & 510 & 505 & 506 \\
Grèce & 504 & 514 & 511 & 488 \\
Espagne & 504 & 497 & 505 & 509 \\
All. Ouest & 503 & 491 & 497 & 520 \\
Colombie & 500 & 502 & 499 & 500 \\
All. Est & 499 & 482 & 493 & 522 \\
Hongrie & 499 & 496 & 493 & 509 \\
Slovénie & 498 & 502 & 489 & 503 \\
Pays-Bas & 485 & 494 & 480 & 481 \\
Chypre & 481 & 492 & 475 & 476 \\
Portugal & 478 & 483 & 480 & 471 \\
Danemark & 475 & 463 & 467 & 496 \\
Trinidad \& Tobago & 451 & 455 & 458 & 440 \\
Indonésie & 394 & 402 & 411 & 369 \\
Venezuela & 383 & 378 & 396 & 374 \\
\hline
\end{tabular}

7 Tous ces résultats montrent que nous n'avons pas à rougir du niveau des élèves français en lecture. Même les élèves les plus faibles (les $5 \%$ qui ont les plus mauvaises notes) ont des scores supérieurs à ceux des autres pays (Finlande et États-Unis exceptés). En France, selon cette enquête, environ $5 \%$ des élèves ont de réelles difficultés de lecture, $20 \%$ sont d'excellents lecteurs. En CM1 plus de $20 \%$ des élèves ont un score supérieur à 18/20 et $50 \%$ ont plus de $15 / 20$. On est donc très loin des chiffres annoncés ici ou là.

D'où viennent ces différences entre les résultats et le sentiment général ? Tout d'abord il n'existe pas de définition de l'illettrisme qui fasse l'unanimité. Personne ne considérera qu'un élève de sixième est illettré parce qu'il ne comprend pas un texte philosophique ou spécialisé. Dans l'enquête internationale, comme nous l'avons dit plus haut, la capacité à lire est définie comme celle de comprendre les formes du langage écrit requises par la société et/ou valorisées par l'individu. Il s'agit donc de lecture fonctionnelle au sens où la lecture se fait sur des textes fréquemment rencontrés: notices, journaux, cartes, histoires. Cette définition n'est pas nécessairement celle utilisée par ceux qui se plaignent de l'illettrisme. La question n'est pas non plus posée de savoir si le niveau du texte donné aux élèves est adéquat.

9 Ensuite, il ne s'agit pas toujours des mêmes populations. Il est certain qu'en France il existe un certain illettrisme "social», que des jeunes ayant eu des difficultés scolaires 
importantes perdent l'habitude de lire et d'écrire et se retrouvent rapidement en état d'analphabétisme ou presque. On ne peut cependant considérer qu'il existe un fort illettrisme "scolaire », mais plus probablement une inadéquation des textes proposés.

10 L'analyse discriminante des textes en lecture ainsi que des réponses des élèves, des enseignants et des chefs d'établissement aux questionnaires d'environnement permet de faire les constats suivants et d'avancer certaines hypothèses expliquant cette contradiction.

\section{Différences entre bons et moins bons élèves de CM1}

11 Les différences entre bons et moins bons élèves sont surtout personnelles et familiales et confirment ce que l'on sait déjà. Le bon élève se juge d'un très bon niveau. Sa famille possède voiture et ordinateur. Passant peu de temps devant la télévision, il lit par plaisir presque tous les jours, même le journal, emprunte des livres à la bibliothèque, mais est peu questionné par ses parents sur ce qu'il lit. Tout se passe comme s'il était autonome.

12 Les moins bons élèves sont âgés. Ils ont leur propre télévision et passent souvent trois heures par jour devant elle et même parfois plus de cinq. Le nombre de livres à la maison est faible. Ils ne lisent presque jamais de bandes dessinées. Mais ces élèves semblent aussi plus aidés par leur famille que les meilleurs. On leur fait la lecture à la maison presque tous les jours. Cette aide ne parait pourtant pas efficace et il est bien possible que cette inefficacité soit provoquée par la distance culturelle entre l'école et la famille. Les parents n'ont peut-être pas connaissance des modèles exigés par l'école. L'école paraît aussi parfois moins exigeante à l'égard de ces élèves : ils ne terminent pas nécessairement leur travail à la maison. En outre, leurs maîtres insistent davantage sur l'apprentissage technique de la lecture que sur le plaisir de lire. L'enfant n'est presque jamais appelé à lire des histoires en classe.

\section{Maîtres et directeurs dans les meilleures et moins bonnes classes en $\mathrm{CM} 1$}

13 L'apprentissage de la lecture étant cumulatif, on ne peut donner à un enseignant ni même à une école la responsabilité de bons ou mauvais résultats dans ce domaine mais il existe cependant des différences d'approche.

Dans les meilleures classes, les instituteurs écoutent souvent leurs élèves lire à voix haute et leur lisent eux-mêmes à voix haute des histoires. Les instituteurs n'ont pas fait d'études à l'université, mais ont suivi de nombreuses heures de formation continue. Leur objectif est de développer l'esprit critique. Ils pratiquent la recherche de thèmes, posent de nombreuses questions et n'insistent pas sur le lexique.

Ces classes se trouvent dans des écoles de dimension moyenne, caractérisées par l'existence de bonnes relations entre les enseignants et les parents. L'acquisition de livres n'est pas nécessairement plus importante qu'ailleurs (10 à 35 ouvrages par an) mais les directeurs ne se plaignent pas du manque de matériel. Ceux-ci ont une grande expérience de l'enseignement. Ce qui caractérise aussi ces écoles, c'est qu'elles ne dispensent pas de programmes spéciaux pour l'apprentissage de la lecture. 
16 Dans les moins bonnes classes, on trouve des enseignants souvent jeunes, qui ne pratiquent presque jamais la lecture silencieuse en bibliothèque et peu à voix haute, qui insistent sur le lexique et ne s'intéressent guère à la pédagogie de la lecture. Peut-être parce qu'ils sont jeunes, ils ont eu peu de formation continue sur ce sujet. Ils n'ont guère d'opinion sur la nécessité d'un suivi des progrès des élèves dans ce domaine.

17 Les directeurs de ces écoles sont eux aussi jeunes (ce sont peut-être les mêmes personnes, puisqu'il s'agit de petites écoles). Les parents coopèrent peu. De nombreux livres ont été ajoutés chaque année à la bibliothèque et surtout il existe des programmes d'amélioration de l'apprentissage de la lecture.

18 Apparemment l'école ne réduit donc pas les inégalités culturelles entre les élèves. Leur niveau en lecture reste largement dépendant du milieu familial.

19 Mais les écoles les plus défavorisées sur le plan des résultats des élèves en lecture sont aussi les plus actives pour résoudre ce problème. Même si la coopération des parents est très moyenne, même si les enseignants ont peu d'expérience, on essaie d'améliorer le niveau, sans pour autant y réussir vraiment. Les résultats seraient peut-être cependant pires si ces programmes n'existaient pas.

20 Toutefois on peut aussi se demander s'il n'y a pas une erreur de stratégie de la part des maîtres et des directeurs d'école. L'accent sur les techniques de la lecture semble important pour les élèves les moins bons. Il ne leur est guère démontré que la lecture est aussi un plaisir, qu'elle peut être ludique et non une « chose » ennuyeuse. Ce n'est pas le manque de livres qui handicape ces élèves et ces classes, c'est le manque de motivation.

21 Ces résultats pour la France se retrouvent de la même façon dans les divers pays. Ceux qui ont un faible niveau en lecture sont aussi ceux pour qui l'apprentissage de la technique est le plus important. Une autre analyse montre que les maîtres des élèves les moins bons ont tendance à augmenter la quantité de travail à faire à la maison et que ceci, ajouté à des programmes spéciaux, peut avoir des effets pervers en renforçant le refus de la lecture chez ces élèves. Il y a aussi une action à entreprendre en direction des parents qui tentent d'aider leurs enfants mais y réussissent mal parce qu'ils ont une mauvaise connaissance de ce que l'école fait dans ce domaine.

\section{RÉSUMÉS}

Dans cet article, l'auteur présente et analyse l'enquête sur la lecture réalisée par l'IEA en 1991, en commentant plus particulièrement les résultats concernant la France et l'influence des stratégies des maîtres en CM1. Il ressort de l'enquête que la motivation apparaît plus importante que la technique. 
INDEX

Index géographique : France

Mots-clés : analyse comparative, enquête, évaluation, lecture, compréhension écrite, comparaison internationale

\section{AUTEUR}

\section{ÉMILIE BARRIER}

Centre international d'études pédagogiques (CIEP) 